\title{
Capsule Commentary on Kroll et al., Benzodiazepines Are Prescribed More Frequently to Patients Already at Risk for Benzodiazepine-Related Adverse Events in Primary Care
}

\author{
Maria E. Garcia, MD MPH \\ Department of General Internal Medicine, Zuckerberg San Francisco General Hospital/University of California, San Francisco, San Francisco, CA, \\ USA.
}

J Gen Intern Med 31(9): 1078

DOI: $10.1007 / \mathrm{s} 11606-016-3766-3$

(c) Society of General Internal Medicine 2016

$\mathrm{T}$ his cross-sectional study by Kroll et al. ${ }^{1}$ reports patient characteristics associated with receipt of benzodiazepines in high and low doses in primary care settings. The authors found that individuals who receive benzodiazepines, particularly at high doses, were more likely to have multiple chronic medical conditions, including a higher likelihood of substance use disorder and depression. These patients were also more likely to have multiple risk factors for adverse events.

It is concerning that patients at higher risk of adverse events from benzodiazepines are more likely to receive these medications; yet, as the authors point out, it is unclear whether benzodiazepines denote higher risk for these patients or whether they are a marker of higher risk patients. It seems unlikely that providers did not realize that these patients were high risk. While reasons for prescribing patterns are unknown, the high complexity and risk factors of these patients suggest that providers may be at a loss for how to treat these patients with complex medical and mental health conditions. In the sample presented, almost half of patients prescribed benzodiazepines were also prescribed antidepressants. Systemic failures, or the lack of good systems in place to treat our most complex patients, ${ }^{2}$ may be contributing to these prescribing patterns. Furthermore, the lack of integration between mental health and primary care may be exacerbating this issue.

One of manifestations of this fragmentation of care is the lack of integration of the electronic medical records ${ }^{3}$ in mental health, primary care and other care systems, which contributes to an inability to track these patients. The authors further found that less than half of the prescriptions were written by the patient's primary care provider, while the rest were prescribed by other providers (who the authors speculate may be specialists, ED clinicians and inpatient clinicians).

While clinicians must play an important role in carefully evaluating prescribing practices and weighing risk factors for individual patients, administrators and policy makers must also work to facilitate the integration of mental health and primary care ${ }^{4}$ and to make electronic medical records available across a variety of care settings.

Corresponding Author: Maria E. Garcia, MD MPH; Department of General Internal MedicineZuckerberg San Francisco General Hospital/University of California, San Francisco, San Francisco, CA 94110, USA (e-mail: Maria.garcia@ucsf.edu).

Compliance with Ethical Standards:

Conflict of Interest: The author has no conflict with any of the material in this manuscript.

\section{REFERENCES}

1. Kroll DS, Nieva HR, Barsky J, Linder JA. Benzodiazepines are prescribed more frequently to patients already at risk for benzodiazepine-related adverse events in primary care. J Gen Intern Med. 2016. doi:10.1007/ s11606-016-3740-0.

2. Smith SM, Soubhi H, Fortin M, Hudon C, O'Dowd T. Managing patients with multimorbidity: systematic review of interventions in primary care and community settings. BMJ. 2012;345, e5205.

3. DesRoches CM, Campbell EG, Rao SR, Donelan K, Ferris TG, Jha A, et al. Electronic health records in ambulatory care-a national survey of physicians. N Engl J Med. 2008;359(1):50-60.

4. Butler M, Kane RL, McAlpine D, Kathol RG, Fu SS, Hagedorn H, et al. Integration of mental health/substance abuse and primary care. Evid Rep Technol Assess (Full Rep). 2008 (173): 1-362. 